\section{Permanent closure of a pancreatic duct leak by endoscopic coiling}
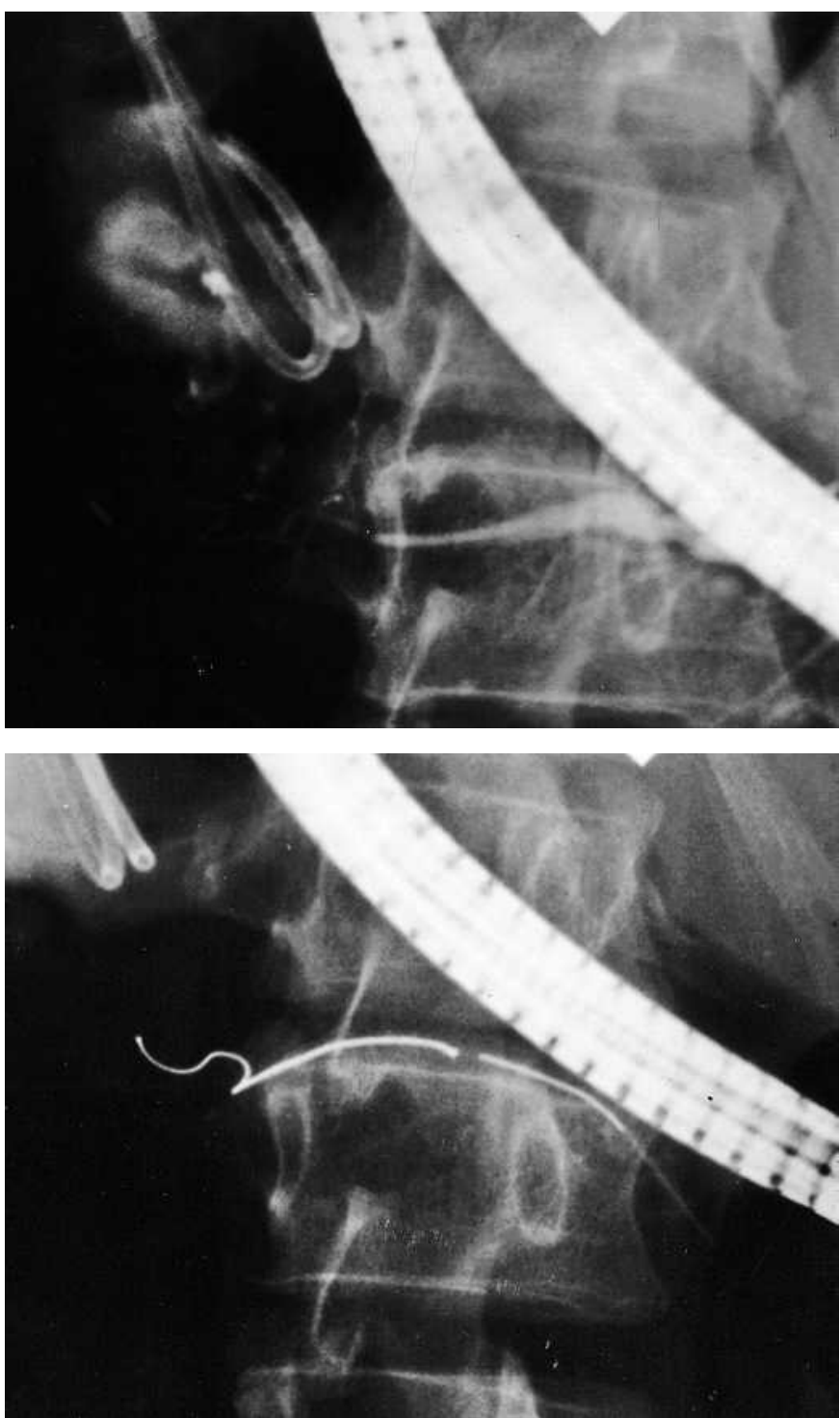

Figure 2 Endoscopic retrograde pancreatographyguided advancement of the coil by pushing with it a nitinol wire. The coil is loaded in a straight position within the catheter and resumes its shape when leaving the tip of the catheter.

A 45-year-old man with a history of chronic pancreatitis was admitted with symptoms of upper abdominal pain. Six months earlier, he had undergone a pancreatic tail resection to remove a suspected tumor (histologically found to be a chronic inflammatory lesion), and a symptomatic cyst (with a diameter of 5 $\mathrm{cm}$ ) ensued locally.

Endoscopic retrograde cholangiopancreatography (ERCP) showed contrast extravasation at the end of the pancreatic duct. Pancreatic endoscopic sphincterotomy and pancreaticoduodenal stent place- ment and conservative therapy failed. Percutaneous drainage was judged to be impossible. An endoscopic ultrasound (EUS)-guided cystogastrostomy [1] was carried out, including balloon dilation and placement of two 7-Fr double-pigtail stents, followed by endoscopic retrograde pancreatography (ERP) to visualize the pancreatic duct and leakage from the end of it (Figure 1). A coil (for intravascular use - a fibered platinum coil, 0.035 inches in diameter, straight length $50 \mathrm{~mm}$, coiled size $5 \times 4 \mathrm{~mm}$; Target Vascular, Boston Scientific, Ireland) was preloaded into an ERCP catheter and then deployed (Fig- ure 2 ) by advancing a 0.035 -inch wire under guidance with fluorescence. In its final position, the coil partly returned to its preset shape and remained firmly in place (Figure 3).

The patient experienced pain relief, and the cyst diameter decreased to $2.5 \mathrm{~cm}$ after 5 days. After 3 weeks, ERP showed complete closure of the leak (Figure 4). Ultrasound and endoscopic examinations of the gastrocystic drain showed that the residual lumen of the cyst was negligible. After removal of the two pigtail stents and an uneventful postinterventional course, the patient was discharged after 26 days. At follow-up examinations after 3 and 12 months, the patient had no further symptoms and a cyst was not evident.

The aim of the standard interventional approach is to drain accumulated fluids $[1,2]$, reduce pancreatic duct pressure, or bypass the fistula/leak [2,3], but these methods may fail. As additional sealing of the leak with glue [4] was deemed inappropriate and risky, we successfully used a vascular coil in the pancreatic duct for this purpose for the first time, on the basis of our experience with transjugular intrahepatic portosystemic stent placement [5]. The coil acts by mechanical obstruction and probably by ductal hyperplasia, due to its fibered surface.

The results were favorable, although it may be difficult to envision the way in which the flow of pancreatic juice might be blocked, despite the rather longitudinal shape of the coil, which was not maximally coiled. In retrospect, it might have been better to use a smaller coil, which would have produced more mechanical obstruction. However, this was a firsttime procedure. Even partial obstruction of a ductal leak (combined with a draining procedure, as described) will promote closure of it. The fibered surface of the coil itself (designed to allow faster clot

DOI: $10.1055 / s-2006-944920$ 


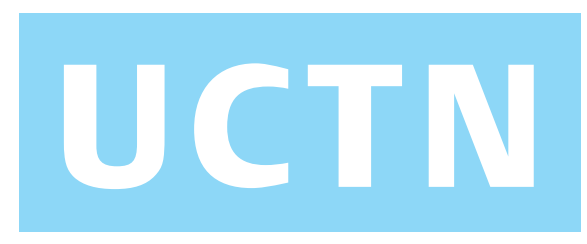

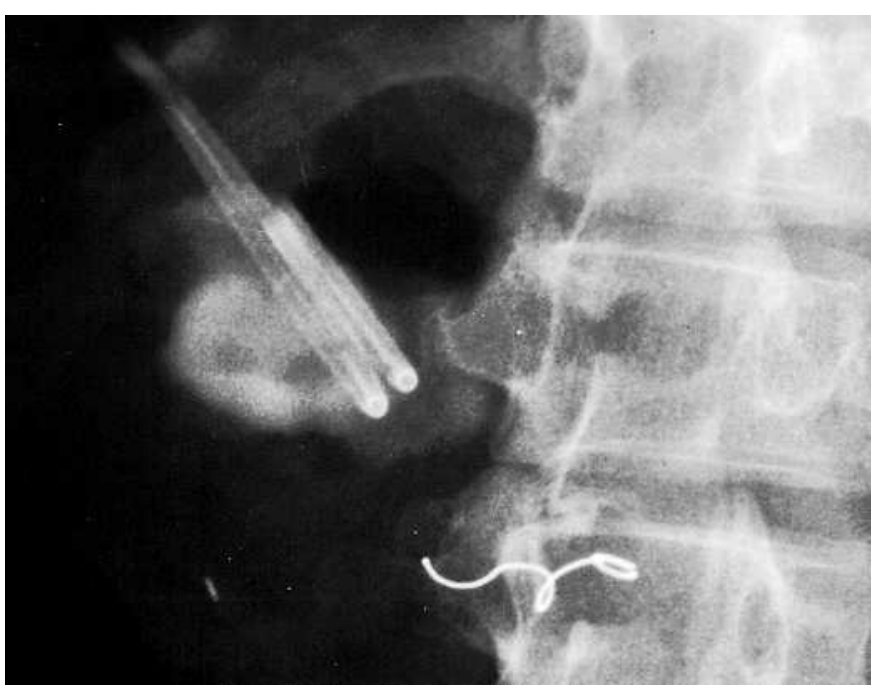

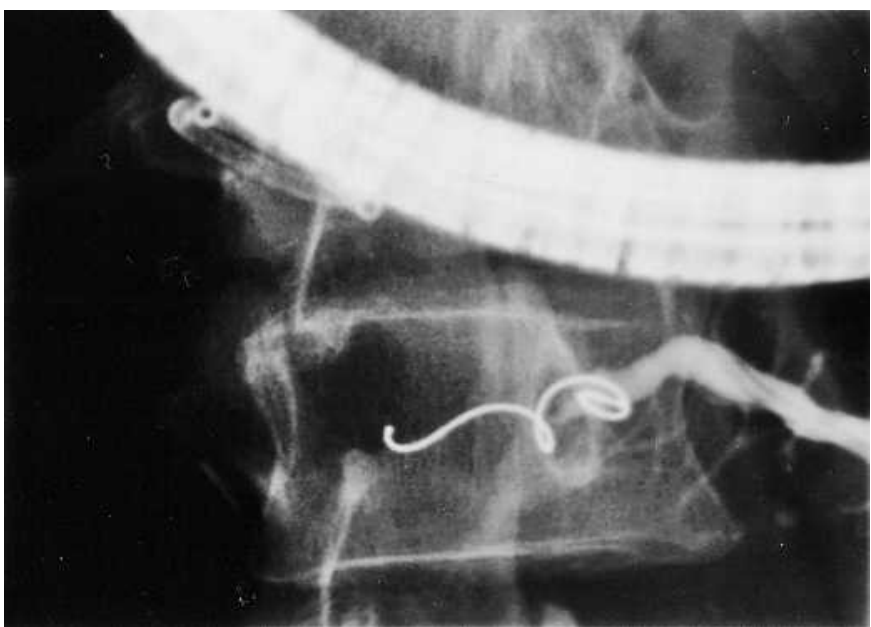

Figure 3 The coil has reached its final shape within the end of the main pancreatic duct. The coil is obstructing the ductal leak.

\section{References}

${ }^{1}$ Fockens P. EUS in drainage of pancreatic pseudocysts. Gastrointest Endosc 2002; 56: S93-S97

2 Costamagna G, Mutignari M, Ingrosso $M$ et al. Endoscopic treatment of postsurgical external pancreatic fistulas. Endoscopy 2001; 33: $317-322$

${ }^{3}$ Kozarek RA, Ball TJ, Patterson DJ et al. Endoscopic transpapillary therapy for disrupted pancreatic duct and peripancreatic fluid collections. Gastroenterology 1991; 100: $1362-1370$

${ }^{4}$ Tran K, van Eijck C, Di Carlo V et al. Occlusion of pancreatic duct versus pancreaticojejunostomy. Ann Surg 2002; 236: $422-428$

${ }^{5}$ Wettstein $M$, Lüthen $\mathrm{R}$, Cohnen $\mathrm{M}$ et al. Transjugular intrahepatic portosystemic stent shunt: indications and long term outcome. Zentralbl Chir 2005; 130: 246-249

Figure 4 The repeat endoscopic retrograde pancreatography ( 3 weeks later) shows complete closure of the previous leak.

\section{Corresponding author}

\section{R. Lüthen, M.D.}

Dept. of Internal Medicine/

Gastroenterology

Marien-Hospital Düsseldorf

(Teaching Hospital of the University

of Düsseldorf) · Rochusstrasse 2

40479 Düsseldorf

\section{Germany}

Fax: +49-211-4400-2591

Email: inneremedizin@ marien-hospital.de formation when applied intravascularly) might also have promoted ductal hyperplasia within the 3-week period before the repeat ERP with contrast injection (Figure 4) definitively confirmed closure of the leak. Further supportive data on this type of coil application will need to be obtained from appropriate animal experiments in vivo and eventually from larger series in humans.

\section{Endoscopy_UCTN_Code_TTT_1AR_2AI}

\section{R. Lüthen', P. Jaklin', M. Cohnen²}

${ }^{1}$ Dept. of Medicine, Division of Gastroenterology, Hepatology and Infectious Diseases, Heinrich Heine University, Düsseldorf, Germany

${ }^{2}$ Institute of Diagnostic and Interventional Radiology, Heinrich Heine University, Düsseldorf, Germany. 